\title{
Apel y la hermenéutica filosófica de Gadamer
}

\author{
Gonzalo Scivoletto
}

CONICET

Resumen: En el presente trabajo se reconstruye el núcleo de la crítica de Apel a la hermenéutica filosófica de Gadamer. Para ello se analizan tres textos de Apel posteriores a La transformación de la filosofía y que por lo tanto van más allá del debate entre hermenéutica y critica de las ideologías. A lo largo del trabajo se intentará mostrar que los conceptos vertidos por Apel pueden abrir la posibilidad de una hermenéutica ilustrada o crítico-normativa.

Palabras clave: comprensión; progreso; entendimiento; Gadamer; Apel

\begin{abstract}
Apel and Gadamer's Philosophical Hermeneutics". This paper seeks to reconstruct the core of Apel's criticism of Gadamer's philosophical hermeneutics. In order to achieve this, three texts post-Transformation of Philosophy by Apel are analyzed. All of them go beyond the debate between hermeneutics and critique of ideology. Throughout the paper I will attempt to demonstrate that the concepts introduced by Apel offer the possibility of an enlightened or critical normative hermeneutics.
\end{abstract}

Keywords: comprehension; progress; understanding; Gadamer; Apel 
El primer contacto de Apel con la hermenéutica de Gadamer se produce a través de la lectura de Verdad y método ${ }^{1}$. Sin embargo, el problema de la comprensión y de las ciencias del espíritu en general no era ajeno a Apel, sino que formaba parte de su formación e incluso es abordado en sus primeros trabajos. La tesis doctoral de Apel, presentada en 1950 y dirigida por Erich Rothacker, se ocupa del problema del conocimiento en Heidegger a través de un prisma kantiano². En 1955, Apel publica un importante artículo sobre el concepto de comprensión ${ }^{3}$ y comienza a trabajar en la elaboración de una historia de la filosofia moderna del lenguaje que pusiera de relieve una tradición "olvidada", en la cual destaca el carácter de apertura de mundo del lenguaje. Esos estudios comienzan con un trabajo sobre Nicolás de Cusa para luego convertirse en el detallado análisis que parte de Dante y concluye en Vico, y que conformará su trabajo de habilitación, donde se acuña el término de hermenéutica trascendental para definir dicha concepción del lenguaje ${ }^{4}$ opuesta a la tradición racionalista y de la búsqueda de un "lenguaje universal" en la línea de Leibniz. Si se tienen en cuenta estos detalles histórico-filosóficos, se puede observar claramente el punto de contacto entre los propios intereses de Apel y esa gran obra fundacional que es Verdad y método5. También Gadamer pone en valor y "rescata" la tradición humanista y de las ciencias del espíritu, poniendo el acento en la importancia de la lingüisticidad de la experiencia. En ese sentido, seguramente la publicación de Verdad y método debió haber despertado en Apel un interés inmediato. Tal vez eso explique un poco, al mismo tiempo, la radicalidad de la respuesta a esa obra que se ubicaba en la tradición y el campo de intereses del cual Apel formaba parte. De hecho, en

1 Una reconstrucción de este encuentro y su impacto la realiza el propio Apel. Cf. Apel, K-O., Auseinandersetzungen in Erprobung des transzendentalpragmatischen Ansatzes, Fráncfort d. M.: Suhrkamp, 1998, pp. 571ss.

2 La tesis mencionada lleva por título Dasein und Erkennen. Eine erkenntnistheoretische Interpretation der Philosophie Martin Heideggers, y se encuentra aún inédita.

3 Apel, K-O., "Das Verstehen (eine Problemsgeschichte als Begriffsgeschichte)", en: Archiv für Begriffsgeschichte, v. I, 1955, pp. 142-199.

4 Apel, K-O., Die Idee der Sprache in der Tradition des Humanismus von Dante bis Vico, Bonn: Bouvier, 1975.

5 Una excelente sintesis de esta primera etapa del pensamiento de Apel, representada por su tesis doctoral Dasein und Erkenttnis y su tesis de habilitación Die Idee der Sprache, se encuentra en Mendieta, E., The Adventures of Trascendental Philosophy, Rowman \& Littlefield, 2002. 
La transformación de la filosofia ${ }^{6}$, Gadamer ocupa, en cuanto interlocutor, un lugar muy destacado.

Sin embargo, la importancia de Gadamer para la filosofia apeliana no se reduce a su etapa formativa y primera sistematización, a saber, desde sus inicios (1950) hasta la publicación de su primera y más conocida gran obra (1973). Por el contrario, en trabajos muy posteriores e incluso recientes, Apel ha seguido teniendo como referente a Gadamer y no solo, como podría pensarse, como objeto de una reconstrucción de sus propias tesis, sino como interlocutor propiamente dicho. Dado que aquellos trabajos posteriores a La transformación de la filosofía no han sido objeto de un análisis detallado en la literatura especializada me propongo aquí analizar tres de ellos, en los que se abordan cuestiones ligadas a la hermenéutica filosófica. Entiendo que tal indagación puede contribuir tanto al análisis de la "historia efectual" de Verdad y método (principal fuente de Apel) como a la historia de la pragmática trascendental, pero más allá de ello puede servir incluso como plataforma para el abordaje de los problemas mismos.

Las referencias y comentarios a la hermenéutica de Gadamer son casi permanentes en la obra de Apel y no hay un abordaje diferenciado por temas o categorías, sino que el lector se encuentra generalmente con posicionamientos sobre el conjunto, cuyas formulaciones reiteradas van abriendo matices diferenciados. Sin embargo, por razones metodológicas y de exposición, creo conveniente seleccionar una serie de textos y abordarlos de manera separada. Los textos a los que me refiero son: el artículo "¿Ideas regulativas o acontecer de la verdad? Sobre el intento de Gadamer de responder a las condiciones de posibilidad del comprender válido"7, un artículo en inglés llamado "Regulative Ideas or Sense Events? An Attemp to Determine the Logos of Hermeneutics"8, y un artículo inédito en alemán pero disponible en una versión inglesa titulado "The Hermeneutics of Being (Heidegger, Gadamer) Versus Transcendental Hermeneutics or Transcendental Pragmatics (Apel)"”.

Apel, K-O., La transformación de la filosofia, Madrid: Taurus, 1985.

Apel, K-O., Semiótica trascendental y filosofia primera, Madrid: Sintesis, 2002, pp. 133-167. El texto original en alemán se encuentra publicado en Apel, K-O., Auseinandersetzungen, pp. 569-607 (en adelante, citado como Ideas regulativas).

8 Apel, K-O., From a Trascendental-Semiotics Points of View, Manchester: Manchester University Press, 1998, pp. 160-182 (en adelante, citado como Regulative Ideas).

9 Publicado en: The Routledge Companion to Twenty Century Philosophy, Londres/Nueva York: Routledge, pp. 763-778 (en adelante, citado como Hermeneutics). 


\section{Comprensión y verdad como ideas regulativas}

El texto "¿Ideas regulativas o acontecer de la verdad?" fue publicado originalmente en 1996, por lo que, si bien en muchos puntos se repiten argumentos de la crítica realizada en La transformación de la filosofía, hay, como decía, nuevos elementos. Lo que primero ha cambiado respecto de la discusión de los años 1960 y 1970, en la que Gadamer participa activamente ${ }^{10}$, es el contexto histórico-filosófico. Por ejemplo, ya no tienen un lugar tan relevante el marxismo y la teoría crítica ${ }^{11}$. De hecho, en este de lapso de veinte años, la crítica de las ideologías ha sido "superada" como programa de investigación dentro de la misma filosofia discursiva de Apel y Habermas ${ }^{12}$. Entretanto, ha cobrado mayor relevancia para Apel, en relación con la filosofia teórica, la cuestión de la destrascendentalización de la filosofia (Rorty, Habermas), las teorías del significado de la tradición analítica y pragmática (Searle, Kripke, Putnam, Lafont), a las que se refiere en diversas ocasiones en el texto, y la arquitectónica de un semiótica trascendental como filosofia primera, la cual también funciona como un punto de partida para la reconstrucción de la historia de la filosofia ${ }^{13}$. En relación con la filosofia práctica, desde fines de los años 1970, Apel se orienta al problema de la parte B de la ética discursiva ${ }^{14}$.

Según Apel, Gadamer convierte los conceptos normativos de la hermenéutica clásica en conceptos "ontológico-temporales" (temporal-ontologische), esto es, en conceptos propios de una teoría del acontecimiento (geschehenstheoretische Begriffe). A saber, comprender como "entrar en el acontecer de la tradición" (Einrücken in das Überlieferungsgeschehen), la aplicación del comprender como "prosecución de la tradición" (Fortbildung der Tradition),

\footnotetext{
${ }^{10}$ Fundamentalmente a través de dos trabajos: "Retórica, hermenéutica y crítica de las ideologías" y la "Réplica a hermenéutica y crítica de las ideologias". Cf., Gadamer, H-G., Verdad y método II, Salamanca: Sígueme, 1998, pp. 251ss.

${ }^{11}$ Sobre este debate, del cual no me ocuparé aquí, cf. Apel, K-O., (ed.), Hermeneutik und Ideologiekritik, Fráncfort d.M.: Suhrkamp, 1971.

${ }_{12}$ Para un intento de rehabilitar dicho programa, con nuevos elementos, ver Bohman, J., "Communication, Ideology and Democratic Theory", en: The American Political Science Review, v. 336 LXXXIV, 1 (1990), pp. 93-109. Una mirada retrospectiva y crítica sobre la crítica de las ideologías la presenta Habermas en "Nach dreißig Jahren: Bemerkungen zu Erkenntnis und Interesse", en: Habermas, J., Erkenntnis und Interesse, Hamburgo: Felix Meiner, 2008, pp. 357-366. Por su parte, Apel presenta, a mi juicio, una posición más ambigua, véase por ejemplo la entrevista realizada por Sáez Rueda en Fernández, D. (ed.), Discurso y realidad. En debate con Karl-Otto Apel, Madrid: Trotta, 1994, pp. 259-260.

${ }_{13}$ Apel, K-O., Paradigmas de filosofia primera, Buenos Aires: Prometeo, 2013.

${ }^{14}$ Al respecto, cf. Apel, K-O., Diskurs und Verantwortung. Das Problem des Übergangs Poskonventionellen Moral, Fráncfort d.M.: Suhrkamp, 1988; The Response of Discourse Ethics, Lovaina: Peeters, 2001.
} 
el círculo hermenéutico como "entrar en juego" (Ins-Spiel-bringen) y "poner en juego" (Aufs-Spiel-setzen) los prejuicios en el modo de la fusión de horizontes ${ }^{15}$. Además, el concepto fundamental de "conciencia de la historia efectual" reúne, según Apel, el concepto de reflexión y el concepto de acontecer (Geschehen). Pero, a diferencia de Heidegger, esta filosofia del ser en cuanto acontecer no es relativa al futuro sino al pasado, a la tradición. De esta característica se derivaría el principio anti-ilustrado de la hermenéutica filosófica de la superioridad del interpretandum. No obstante, Apel aclara que no se encuentra en contra de la concepción ontológico-temporal del comprender, sino que lo que pone en tela de juicio es si tal concepción es suficiente para entender o conceptualizar la corrección (Richtigkeit) o profundidad (Tief) del comprender: "Precisamente, en tanto que determinación ontológico-temporal [el principio de comprender siempre diferente], se refiere tan solo a las precondiciones de todo intento humano de comprender, pero no a las condiciones trascendentales de posibilidad del comprender válido o no válido. Estas, por su naturaleza, se refieren contrafáctica e intemporalmente a la capacidad universal de consenso de todo intérprete concebible"16.

Tal principio, como idea regulativa, se encuentra implícitamente en todo comprender creativo: se trata del comprender reflexivo superador (reflexiv überholendes Verstehen). Para aclarar esto, Apel menciona un ejemplo extraído del propio Gadamer, donde este se refiere al "progreso" (aunque Gadamer no utiliza ese término) efectuado por Heidegger respecto de la metafísica moderna: “...en la prueba heideggeriana, citada por Gadamer, de que en los conceptos de conciencia en Descartes y de espíritu en Hegel sigue dominando la ontología griega de la sustancia... y que darse cuenta de ello '(conduce) más allá de la autocomprensión de la metafísica moderna'. ¿Cómo se compadece esta afirmación de Gadamer con su recusación por principio del 'comprender mejor'?"17.

La posibilidad de un comprender mejor no es simplemente una afirmación dogmática o arbitraria, sino que es la consecuencia que se deriva de la "simetría del entendimiento dialógico (dialogischen Verständigung)"18, que también es un presupuesto fundamental de la hermenéutica filosófica. Como mostraré más adelante, para Apel los dos pilares o principios sobre los que se sustenta la

\footnotetext{
15 Apel, K-O., Ideas regulativas, p. 135.

16 Ibid., p.137.

17 Ibid., p. 138. La cita de Apel está extraída de Gadamer, H-G., Verdad y método, p. 336.

18 Apel, K-O., Ideas regulativas, p. 139.
} 
hermenéutica se encuentran, como mínimo, en tensión: el principio del acontecimiento y el principio dialógico.

Apel entiende que hay dos "presupuestos problemáticos" en la hermenéutica de Gadamer, a saber: la teoría de la verdad como aletheia y la equiparación (Gleichsetzung) de la exigencia de la validez intersubjetiva del "comprender mejor" con el ideal de objetividad de las ciencias naturales. Según Apel, el presupuesto, necesario pero no suficiente, de la apertura de sentido del mundo de la vida le proporcionó a la hermenéutica filosófica de Gadamer el concepto de verdad que necesitaba. La comprensión hermenéutica no tiene que ver con el conocimiento de cosas intramundanas (Erkenntnis von innerweltlichen Dingen), sino con la manifestación del sentido (Offenbarkeit von Sinn), por ejemplo, el sentido lingüístico en la experiencia cotidiana o de obras de arte y textos. Por lo tanto, confiesa Apel, siempre le pareció poco claro "cómo una hermenéutica que se refiere únicamente al sentido comprensible (verstehbaren Sinn) podría alcanzar... el estatus de una filosofia universal del entendimiento intersubjetivo sobre el mundo y sobre la cognoscibilidad de entes intramundanos"19. Desde el punto de vista de Apel, una teoría de la verdad como aletheia solo sería suficiente si pudiera abstraerse metodológicamente el sentido comprendido de la evaluación del intérprete, es decir, si pudiera separarse la comprensión del sentido (Sinn-Verstehen) del entendimiento valorativo (wertenden Verständigung). Por "valorativo" debe entenderse aquí el "enjuiciamiento" sobre las pretensiones de validez del interpretandum ${ }^{20}$. Es decir, es imposible comprender el "sentido en sí" de un texto sin ingresar en un "discurso virtual" con el autor que expresa, mediante el texto, determinadas pretensiones de validez ${ }^{21}$. Precisamente es el propio Gadamer el que combate el objetivismo de la hermenéutica clásica, con lo cual debería haber aquí un punto de convergencia. De hecho, tanto Apel como Gadamer parten del paradigma del Verständigung über etwas, por lo que, para ambos "comprender el sentido (Sinnverstehen) y alcanzar un acuerdo (Einverständnis) sobre algo van juntos en el mundo de la vida"22.

19 Ibid., p. 143. Destacado en el original.

${ }^{20}$ En el caso de un texto, de las pretensiones de validez de un autor respecto de la cosa sobre la que se discute. Por supuesto que aquí nos encontramos con una relación derivada y en cierto punto deficitaria del modelo del diálogo actual, es decir, cuando dos interlocutores discuten efectivamente pretensiones de validez hic et nunc, puesto que en el caso de la interpretación de textos generalmente el autor no puede responder. La caridad hermenéutica puede interpretarse en este sentido como un pre-requisito para la evaluación de pretensiones de validez.

${ }^{21}$ Para un análisis más detallado de la posición apeliana aplicada a la hermenéutica textual, ver Böhler, A., "Philosophische Hermeneutik und hermeneutische Methode", en: Fuhrmann, M. y otros (eds.), Text und Applikation, Múnich: Wilhelm Fink, pp. 483-511.

${ }^{22}$ Apel, K-O., Ideas regulativas, p. 143. 
Ahora bien, aquí aparece, a mi modo de ver, un elemento problemático. Según Apel, no se puede restringir la referencia a la cosa (Sachreferenz), a la aperturidad de sentido (Sinnerschlossenheit) del mundo. La posibilidad, presupuesta por Apel con Putnam y Kripke, de corrección del significado mediante la referencia permite introducir una dimensión de la verdad que sería dejada de lado por la concepción de la verdad como aletheia, esto es, la adecuación o corrección (Richtigkeit). La posición de Apel en este punto no ofrece ninguna duda. Luego de una descripción de cómo las extensiones pueden corregir las intensiones conceptuales, Apel afirma categóricamente: "De este modo, se corrige la tesis de Heidegger de la diferencia ontológica rígida; o dicho con otras palabras: la tesis de que es la comprensión del ser la que abre el ámbito de juego para el conocimiento óntico-empírico"23. Después, volviendo a Gadamer, aclara: "Pues por mucho que la manifestación fáctica del sentido (la aletheia en el sentido de Heidegger) sea siempre relativa a cada situación, la verdad objetiva ${ }^{24}$ de las pretensiones de validez no está, en cualquier caso, suficientemente determinada por la manifestación fáctica del sentido. Antes bien, enjuiciarlas supone una evidencia (Einsicht) ${ }^{25}$ que tiene que ser válida contrafácticamente, esto es, válida para una comunidad ilimitada de interpretación. Y si esta explicación del sentido de la verdad tiene en general algún sentido pragmático, tendríamos por principio que poder suponer la posibilidad de progresos cognoscitivos, por de pronto en la dimensión de la adecuación objetiva (sachbezogenen Richtigkeit)... Esto significa que el contar con posibles progresos cognoscitivos tiene que ser constitutivo del mundo de la vida, ya para la comprensión lingüisticamente mediada, pero además también para el comprender en el sentido estricto, metodológico, de la hermenéutica propia de las ciencias del espíritu"26.

Frente a esta defensa de Apel de la posibilidad de comprender mejor, de corregir el sentido de acuerdo con la investigación sobre la cosa, podría objetarse que tal modelo se orienta por una concepción objetivista, progresiva

\footnotetext{
23 Ibid., p. 145.

24 Aquí la traducción de sachbezogen Wahrheit por "verdad objetiva" puede prestarse a confusión. Se trata de la verdad "relativa a la cosa" sobre la cual se plantean las diferentes pretensiones de validez. Es decir, aquello sobre lo que se discute, la cosa, aun cuando no se llegue a un consenso sobre ello. "Verdad objetiva" puede sugerir la idea de una verdad última, incuestionable, y no es esto a lo que Apel se está refiriendo. La misma confusión se genera con la expresión de más abajo: "adecuación objetiva".

${ }^{25}$ El término Einsicht tal vez debería ser traducido como "intelección" o "comprensión" (en inglés, "insight" o "comprehension"). Es importante esta aclaración porque la evidencia, para Apel, es un "momento" necesario de la teoría, criteriológicamente relevante, de la verdad. En tal caso utiliza el término Evidenz.

26 Ibid., p. 146
} 
e incluso "cientificista". La respuesta a esta crítica posible nos introduce en el segundo presupuesto de la hermenéutica filosófica, que consiste, según Apel, en la equiparación de la validez intersubjetiva del progreso de la comprensión con la objetividad de la ciencia natural progresiva.

De acuerdo con Apel, la imposibilidad del "comprender mejor" en Gadamer se debe a la "inexistencia", por así decirlo, de un objeto sobre el cual pueda realizarse una investigación progresiva. Es decir, aunque se distinga entre la manifestabilidad del sentido (Sinn-Offenbarkeit) y la adecuación relativa a la cosa (sachbezogener Richtigkeit), y la estructura de la comprensión hermenéutica (que se encuentra a la base de toda experiencia del mundo, también la científica) tenga la forma de un "entendimiento sobre las cosa" (Verständigung über etwas), según Apel, "para el comprender hermenéutico no puede haber referencia objetiva (Sachreferenz) alguna que permita un progreso del conocimiento"27. Sin embargo, precisamente la hermenéutica filosófica de Gadamer, como reconoce también Apel, pone el acento en la cosa. Parece entonces que la "cosa" es para Gadamer y Apel algo diferente. Sigamos con la respuesta de Apel. En primer lugar, aclara que él no comprende el ideal de objetividad (Objetktivitätsideal) de la ciencia natural como modelo para la referencia objetiva de la comprensión hermenéutica. Es decir, que no entiende por "cosa" del entendimiento el "objeto" de las ciencias, el cual como objeto ideal se encuentra "acabado" (fertig) y es, por esa razón, susceptible de un conocimiento progresivo. Apel concede entonces a la hermenéutica filosófica que en las ciencias del espíritu la referencia objetiva, esto es, la cosa, acontece a través de la mediación de la historia y la tradición que ellas mismas ayudan a proseguir. Pero, a pesar de esto, para Apel todavía es posible pensar un progreso de la comprensión en las ciencias del espíritu. Para ello hay que distinguir entre la validez intersubjetiva (intersubjektiven Gültigkeit) y la "objetividad" en el sentido de las ciencias naturales. Un ejemplo de validez intersubjetiva "no objetiva" lo representan las normas éticas que guían la propia comprensión hermenéutica. Sin embargo, aquí se presenta una dificultad, en la medida que Apel evade el problema de la comprensión propiamente dicho. Es decir, a mi modo de ver, independientemente de la posibilidad de fundamentación de normas morales intersubjetivamente válidas ("objetivas"), el problema de la comprensión hermenéutica y su posible conocimiento sigue quedando abierto en Apel. En efecto, si la comprensión hermenéutica se aboca a la tarea de comprender las expresiones de sentido, según la cual el texto representa un

27 Ibid., p. 148. 
modelo paradigmático, no termina de quedar claro de qué manera "la cosa", siempre ya mediada por el acontecer histórico (como reconoce el propio Apel), sea susceptible de un conocimiento progresivo sin abstraerla precisamente de dicho acontecer. En otras palabras, no termina de quedar claro cómo sería posible comprender mejor un texto sin presuponer al mismo tiempo que dicho texto tiene una estructura "real", homogénea e invariable, de modo similar al objeto de las ciencias naturales. Ahora bien, la reflexión de Apel no se dirige al estatuto ontológico del objeto de las ciencias del espíritu, sino a las condiciones intersubjetivas de posibilidad del conocimiento, esto es, al discurso argumentativo. El principio del comprender mejor, por tanto, no se deriva de la estructura de la cosa, sino que se muestra, mediante reflexión trascendental, como un presupuesto necesario del discurso.

El discurso argumentativo posee una doble característica: es, al mismo tiempo, la forma reflexiva del entendimiento sobre algo y la condición normativa para un comprender hermenéutico crítico. En este punto, hay una rectificación fundamental de Apel. En una mirada retrospectiva al debate entre hermenéutica y crítica de las ideologías, sostiene: "Habermas y yo sostuvimos -tal como hoy lo veo, sin razón- que la 'pretensión de universalidad' de la hermenéutica filosófica de Gadamer podía ponerse en cuestión desde enfoques propios de las ciencias sociales... Dicho con mayor precisión, desde la mostración de la posibilidad y necesidad de suspender crítico-reflexivamente la 'anticipación de la perfeción' hermenéutica por medio de la crítica objetivante de la competencia comunicativa de los seres humanos socializados. Sin embargo, tanto Habermas como yo llegamos a desarrollar simultáneamente una filosofia discursiva que mostraba la imposibilidad de tal puesta en tela de juicio"28.

Esta imposibilidad de poner en tela de juicio la pretensión de universalidad de la hermenéutica está supeditada a que no solo se plantee la pregunta por las condiciones de posibilidad de la comprensión, sino también por las condiciones del comprender válido, esto es, en el sentido de una hermenéutica trascendental. En cuanto a la crítica de las ideologías, con tal afirmación Apel no pretende restarle valor o importancia como herramienta para la crítica social. Podría decirse que la filosofia discursiva funciona, si se me permite el término, como una metafilosofia, en cuanto analiza la estructura del discurso presupuesta por todo "discurso" científico o filosófico social. Así, por ejemplo, los "discursos" psicoanalítico, genealógico, crítico de las ideologías o decons-

${ }^{28}$ Ibid., p. 151. 
tructivista, etcétera, son formas específicas de ejercicio filosófico que pueden funcionar con relativa autonomía y eficacia en la medida que sus postulados no contradigan los presupuestos del discurso argumentativo en general, presupuesto como condición de posibilidad y validez de su propio campo. Al fin y al cabo, la actividad científica no consiste más que en la discusión, mediante razones, sobre la corrección de una interpretación o teoría, o de la efectividad de tal o cual procedimiento, etcétera. Que no pueda ponerse en tela de juicio la pretensión de universalidad de la hermenéutica significa que cada "discurso" posee la estructura del entendimiento comunicativo, basado en el lenguaje común que constituye su respectivo campo semántico ${ }^{29}$. El discurso argumentativo, en tanto forma reflexiva de tal entendimiento, presenta las condiciones de sentido de cualquier "discurso". Siempre que nos movamos en el plano del discurso argumentativo, los "discursos" particulares que expresan perspectivas históricamente condicionadas adquieren una forma reflexiva. Una vez que adquieren tal forma, aparece la posibilidad de evaluación crítica y, con ello, de "acceso a nuevas y superiores perspectivas comprensivas, es decir: de nuevas formas de la precomprensión del mundo que posibilitan un comprender más profundo y que no son meras 'prosecuciones' de tradiciones, sino más bien logros de la reflexión crítica"30. Se generan así, mediante la reflexión crítica, nuevas "aperturas de sentido". Tales aperturas de sentido, reconoce Apel, son finitas y situadas debido a que no presentan la característica de los juicios reflexivos filosóficos (trascendentales), a saber, la ausencia de un "punto de vista" material. Ahora bien, uno podría preguntarse por qué tales nuevas comprensiones o aperturas de sentido son "más profundas" o "mejores". ¿Cómo podemos tener "certeza" de ello? Creo que planteado de esta manera se realiza una jugada incorrecta, en la medida que se traslada el principio del comprender mejor del lado del sujeto al lado del objeto, por utilizar un lenguaje clásico. En todo caso, podría plantearse un "progreso" en el plano del discurso reflexivo, que consiste precisamente en una estructura formal carente de contenido. Sin embargo, ello llevaria a sostener que el discurso reflexivo-formal sobre los discursos resultaría improductivo o irrelevante para los discursos de contenido y abridores de mundo. Ahora bien, Apel se opone rotundamente a tal afirmación y muestra

\footnotetext{
${ }^{29}$ Lo mismo sucedería, según Kuhlmann, con la ética discursiva en relación con otras teorías éticas (utilitarismo, ética de la virtud, ética del cuidado, ética de los valores, etcétera). $C f$., Kuhlman, W., "Una nueva imagen de la ética filosófica. ¿Tiene la ética discursiva una posición especial en la ética filosófica?", en: Michelini, D. y otros, Ética, politica y discurso, Ediciones del ICALA, 2015, pp. 29-39.

${ }^{30}$ Apel, K-O., Ideas regulativas, p. 155.
} 
cómo los presupuestos del discurso, descubiertos por reflexión trascendental, "ponen en libertad" nuevas comprensiones, por ejemplo acerca de las normas morales universales o de las formas mismas de reconstrucción histórico-critica de las ciencias hermenéuticas. En otras palabras, la "prueba" del principio del comprender mejor la aportaría precisamente la misma filosofia discursiva que ha logrado mostrar el a priori irrebasable del discurso argumentativo como forma reflexiva de todo entendimiento, tanto en el mundo de la vida como en cualquier discurso científico.

La "reflexión", sobre la que carga Gadamer, como veremos enseguida, no puede ser entendida en un sentido psicológico. La reflexión es la capacidad virtual de cualquier interlocutor de, gracias a la estructura del lenguaje, "volver" sobre las normas implícitas presupuestas cuando formula una determinada pretensión de validez. En este sentido, cada interlocutor se encuentra, por decirlo así, sujeto a una doble facticidad. Por un lado, a la facticidad descripta por la hermenéutica de la pre-estructura de su ser en el mundo, su precomprensión expresada en un lenguaje público que lo precede y lo constituye. Por otro lado, al mismo tiempo se encuentra sujeto a la facticidad de la "pre-estructura" de la reflexión sobre esa precomprensión o cotidiano ser en el mundo. Dicho de una manera un tanto simple, un interlocutor que participa en un discurso es un sujeto concreto, perteneciente a una cultura o forma de vida concreta y que "habla" con un lenguaje específico (es decir, utiliza un lenguaje público), pero al formular determinadas pretensiones de validez puede "volverse", mediante el mismo lenguaje público, y analizar los presupuestos necesarios, éticamente relevantes, de su discurso actual. Ahora bien, lo interesante de este análisis de Apel reside en que tales presupuestos pertenecen "hoy también al a priori fáctico de nuestro ser en el mundo" ${ }^{31}$. En esto consiste el denominado principio de autoalcance (Selbsteinholungsprinzip) de la reconstrucción histórica, el cual representa para Apel una alternativa tanto a la filosofia de la historia metafísico-dogmática como a la hermenéutica del acontecimiento de sentido: "El reconocimiento de las mencionadas presuposiciones no solo constituye, para todas las ciencias crítico-reconstructivas, un a priori de la argumentación no discutible, sino que también representa para ellas un dato de la historia a reconstruir, un dato que solo a través de la reconstrucción comprensiva de la historia, según su posibilidad y efectividad puede ser, por así decirlo, 'recogido' o alcanzado. El punto de partida hermenéutico trascendental que presupone

${ }^{31}$ Ibid., p. 165. 
la reconstrucción histórica hace, pues, también las veces de telos de la reconstrucción, cuya función como principio regulativo no puede ser discutida, si es que la reconstrucción no quiere caer en contradicción performativa con sus propias condiciones de validez"32.

Apel entiende este modelo "comprensivo" de reconstrucción histórica como opuesto a las "explicaciones", que considera (cuasi) causales, por ejemplo, la genealogía de Nietzsche, y lo encuentra análogo a la idea de reconstrucción racional de Lakatos, como un ensayo de buenas razones en la historia interna de la ciencia. Pero Apel no solo defiende el modelo de reconstrucciones racionales para la historia de la ciencia, sino que lo expande a la "reconstrucción de la historia del espíritu o de la evolución de la cultura en su totalidad"33, por ejemplo, de la moralidad y del derecho. En su libro Paradigmas de filosofia primera, Apel presenta análisis concretos o casos como muestras de "intentos de reconstrucción”. En este intento de recuperar el concepto de progreso, además de Kant, Apel vuelve a uno de los filósofos que había sido objeto de estudio en sus inicios: Giambattista Vico. Lamentablemente no puedo desarrollar aquí la lectura apeliana de $\mathrm{Vico}^{34}$, pero valga al menos como un símbolo para pensar la relación Apel-Gadamer.

\section{El progreso en cuestión}

El concepto de progreso y su defensa por parte de Apel es sin duda uno de los puntos poco simpáticos o políticamente incorrectos en nuestro horizonte filosófico contemporáneo. Sin embargo, creo que precisamente desde un punto de vista hermenéutico es posible poner a trabajar nuestros propios prejuicios sobre (o contra) la idea de progreso. El segundo texto sobre el que me quiero detener, "Regulative Ideas or Sense Events? An Attemp to Determine the Logos of Hermeneutics", puede ser un puntapié inicial para dicha tarea. Allí Apel afirma: "Se debe enfatizar que por supuesto no es necesario sostener que la historia del mundo o de la humanidad tiene un 'curso necesario' (necessary path) o tiene que ser concebida o profetizada como un proceso determinado

344 causal o teleológicamente. Este meta-récit de la modernidad (Lyotard) tal como es desplegado por Hegel, Comte o Marx bien puede ser declarado muerto"35.

\footnotetext{
${ }^{32}$ Ibid.

${ }^{33}$ Ibid., p. 167.

${ }^{34}$ Respecto de la lectura apeliana de Vico, cf. Apel, K-O., Die Idee der Sprache, pp. 318-380; Paradigmas de filosofia primera, pp. 219-254.

${ }^{35}$ Apel, K-O., Regulative Ideas, p. 161.
} 
Sin embargo, para Apel, antes de esta "hybris", como él mismo la llama, Kant habia introducido un concepto de progreso completamente diferente. Aquí, el progreso no es una "ley de la historia" sino que consiste en el "deber moral" de tener como posible tal progreso, incluso resistiendo a los fracasos. Se trata de un progreso en el ámbito de la praxis, concretamente la moral, que es donde Apel pone el acento en este artículo, esto es, en la relación entre hermenéutica y filosofia práctica. Precisamente, para Apel, si bien la hermenéutica de Gadamer no se desentiende de la problemática de la obligación moral, la desvincula de la idea regulativa de un progreso universalmente válido y de evaluaciones (judgments) de pretensiones de validez para ubicarla exclusivamente en el ámbito de un "nosotros", en "prácticas de una forma de vida" (Wittgenstein). En este punto, Apel realiza una breve comparación entre la hermenéutica y el pensamiento "posmoderno" de la "crítica total a la razón" y utiliza como ejemplo de tal crítica total a Derrida ${ }^{36}$.

Para Apel, Derrida quiere abandonar toda atadura de la metafísica occidental con su "logos", y es en tal sentido un continuador de la destrucción de Heidegger, ahora mediante la deconstrucción. Pero, por otra parte, intentaría ir más allá de Heidegger y pensar la diferencia ontológica como el “... juego infinito de la 'diferencia', que libera el sentido simbólico, pero al mismo tiempo lo 'desplaza' (displace), de tal modo que solamente el juego infinito de la 'diseminación' de los significantes siguen siendo para nosotros -el siempre infructuoso intento, por decirlo así, por descubrir y arrancar (root out) el 'rastro' (trace) del significatum trascendental..."37.

No obstante, más adelante, Apel agrega: "De buen modo puede uno admitir que los procesos de conocimiento humano, en cuanto procesos de interpretación sígnicamente mediado (sign-mediated) de signos, son como tales empíricamente incompletos, $\mathrm{y}$, debido a la diferencia constitutiva de sentido entre el acto singular del uso del signo y el modelo repetible [la iteración] de

\footnotetext{
${ }^{36}$ Las relaciones entre la hermenéutica y el deconstruccionismo exceden ampliamente los límites de este trabajo. Según Grondin, Gadamer consideró como "excelente" la crítica que Habermas realiza a Derrida (Grondin, J., Introducción a la hermenéutica filosófica, Barcelona: Herder, 1999, p. 192, nota 430), critica que en lo fundamental coincide con la de Apel. De manera muy resumida puede decirse que el núcleo de esa crítica se basa en la acusación de autocontradicción performativa de Derrida al intentar poner en cuestionamiento la comunicación y la comprensión, o la argumentación en general, precisamente, argumentando. Al argumentar contra la argumentación se presuponen in actu las reglas contra las que se argumenta. Para un análisis de la idea de "crítica total a la razón", ver Scivoletto, G., "Hacia un nuevo humanismo: Karl-Otto Apel y la crítica total a la razón”, en: Arpini, A. (comp.), El humanismo, los humanismos, Mendoza: Ediunc, 2015, pp. 141-151.

${ }^{37}$ Apel, K-O., Regulative Ideas, p. 163.
} 
la forma significante, están sujetos a un infinito acontecimiento-juego de la différance de sentido diferencial y de la diseminación" 38 .

A pesar de este reconocimiento, Apel presenta dos objeciones. En primer lugar, entiende que no es necesario suprimir la idea de un significatum trascendental, sino que más bien este debe ser entendido en un sentido peirceano como "interpretante último", esto es, no como un hecho alguna vez alcanzable de manera absoluta sino como una idea regulativa (presupuesta en la argumentación). En segundo lugar, Apel insiste en que es necesario un acuerdo intersubjetivamente válido sobre el sentido en el discurso actual, es decir, por ejemplo, en el momento que Derrida eleva su pretensión de validez para siquiera "poder pensar" y "comunicar" tales pretensiones.

Finalmente, otro de los aspectos relevantes en este artículo es la aclaración del rol que cumple el principio de autoalcance como principio metodólogico alternativo a una filosofia especulativa de la historia y a la negación "nihilista" de todo principio. El principio de autoalcance tiene la forma del "círculo hermenéutico", con lo cual Apel coincide con la idea fundamental de la hermenéutica desde Heidegger de que todo conocimiento (comprensión) de la realidad debe tomar como punto de partida su propia situacionalidad y, también con Heidegger, Apel cree que no se debe querer "evitar" el círculo sino ingresar "de modo correcto" en él. Ahora bien, ¿qué significa "ingresar correctamente" en el círculo de la comprensión? Para responder a esta pregunta, Apel vuelve sobre el concepto gadameriano de "anticipación de la perfección" y dice: "El 'preconcepto de perfección' (Gadamer) con respecto al juicio evaluativo del interpretandum es válido en la medida que el intérprete encuentra razones para ser capaz de comprender las pretensiones de validez del interpretandum en el sentido de las pretensiones diferenciadas de validez universal del lenguaje humano en tanto bien fundadas" 39 .

Todo proceso de comprensión empírica, del cual la interpretación de textos puede funcionar como paradigma, supone que el lector/intérprete comprende y evalúa las pretensiones de validez presentadas en dicho texto. En cuanto pretensiones de validez, además, poseen la triple referencia a un mundo objetivo, social e "interno" (Habermas), y por lo tanto "pueden ser evaluadas como posibles contribuciones a una historia progresiva interna" del conocimiento, de juicios normativos (derecho, moral) o del arte en general como autoexpresión sincera o auténtica. La "evolución cultural" (obviamente no en un sentido teleológico, sino

38 Ibid., p. 172.

39 Ibid., p. 178. 
en un sentido weberiano-lakatoniano) puede ser entendida como un proceso de aprendizaje autosostenido de largo plazo (long-term self-sustaining process of learning), que puede ir introduciendo "correcciones" o "modificaciones", esto es, que alteran progresivamente la "apertura de mundo" o la constitución previa de sentido de una forma de vida entrelazada con el lenguaje natural. Ahora, si bien creo que aún queda por precisar más detalladamente en qué consiste el círculo hermenéutico entre apertura de sentido y procesos de aprendizaje, al menos tenemos una indicación de cierta cercanía de Apel con Gadamer en cuanto a la "preeminencia de la pregunta", es decir, en cuanto al postulado hermenéutico fundamental de que toda afirmación o proposición es una respuesta a una pregunta, y que precisamente esa pregunta se encuentra determinada por una situacionalidad o facticidad: "sobre esto, parece que la apertura de mundo es decisiva a priori”"40. Apel llega incluso a afirmar que el asunto o tema (issue) para una reconstrucción hermenéutica de la evolución de la cultura es qué cuestiones pueden ser planteadas en su cultura y cuáles no. A mi juicio, esta forma de reconstrucción hermenéutica crítico-normativa es aplicada por Apel a la hora de reconstruir la ética discursiva misma, esto es, como una especie de dialéctica entre "desafios externos" y "recursos internos". El proceso de aprendizaje se ve "impulsado" por desafios históricos, por una situacionalidad concreta, es decir, aquello que "abre" una pregunta. En el caso de la ética discursiva, por ejemplo, la pregunta por la posibilidad de una moral universalmente válida frente a la "catástrofe moral" del nazismo. Sin embargo, ¿en qué medida la evolución de las concepciones práctico-morales, en otras palabras, de la ética, puede considerarse como "progresiva"? ¿Representa la ética discursiva el fin de la historia de la ética, frente a la cual, todas las teorias anteriores no son más que una mera pre-historia? Y si nos trasladamos al campo teórico empírico o científico, ¿̇en qué medida puede considerarse que la historia del conocimiento sigue un conocimiento progresivo? El modelo de reconstrucciones racionales de Lakatos, al cual Apel se adhiere, ¿no presupone ya que dicha reconstrucción se realiza en el marco de un mundo ya abierto, digamos, el mundo "occidental"? Ahora bien, y esto lo reconoce el propio Apel, más problemático aun es el campo estético donde dificilmente pueda hablarse de algo así como "progreso". Por último, queda el ámbito de la filosofia misma. También aquí Apel es de la idea de una evolución histórica progresiva de paradigmas de filosofia primera, donde la semiótica trascendental subsume, por decirlo así, los paradigmas ontológicos y de la conciencia.

${ }^{40}$ Ibid., p. 179. 


\section{3. ¿Entendimiento versus Acontecimiento?}

El tercer y último texto que analizaré aquí es mucho más reciente. Se trata de un artículo inédito en alemán, pero del cual existen, hasta ahora, dos traducciones publicadas, una en italiano y otra en inglés. Aquí me baso en la versión inglesa publicada en una enciclopedia de filosofia contemporánea. El título original del trabajo es Seinshermeneutik (Heidegger und Gadamer) versus 'Transzendentalhermeneutik' bzw. 'Transzendentalpragmatik'(Apel).

En este texto, Apel comienza señalando un aspecto (implícitamente positivo) que diferencia a Gadamer de su maestro Heidegger. Se trata de la relación con la historia de la filosofia. Frente a la "destrucción" (deconstrucción) de Heidegger, por ejemplo, de Platón y Aristóteles como fundadores de la metafísica, Gadamer lleva a cabo una tarea de diálogo y "rescate"41. En este sentido, para Apel hay dos motivos o dos orientaciones de la hermenéutica filosófica de Gadamer: el enfoque heideggeriano y el modelo dialógico socráticoplatónico. En cuanto al primer punto, Apel señala que la hermenéutica parte de la "identificación del comprender (Verstehen) con el modo de ser temporal de la existencia humana"42 y que, por lo tanto, la interpretación del sentido no puede convertirse en un método puramente objetivo, ya que es una "práctica subjetiva" de aplicación en el mundo de la vida. El segundo aspecto es el más relevante en nuestro contexto, dado que permite establecer un puente entre la hermenéutica filosófica y la filosofia discursiva de Apel. Gadamer parte de la situación dialógica, esto es, del entendimiento entre dos interlocutores sobre algo, aun cuando la interpretación de textos (el diálogo entre un intérprete y un interpretandum) no sea completamente equivalente a una conversación "real". En otras palabras, la pregunta fundamental que se podría plantear a la hermenéutica de Gadamer es cómo es posible conciliar el punto de partida de una filosofia del acontecimiento ("acontecer temporal del ser") con una filosofia dialógica cuyo punto de partida es el "entendimiento a través del diálogo"43. No obstante, ¿por qué ambos puntos de partida deberían ser considerados como irreconciliables?

348 El primer punto que señala Apel es la limitación que posee el concepto de "fusión de horizontes". Es decir, aunque se concediera que la fusión de horizontes es una precondición ontológica del proceso de comprensión (compren-

\footnotetext{
${ }^{41}$ Como el propio Gadamer señala en numerosas ocasiones, esto es muy evidente en el caso de la interpretación sobre Platón.

${ }^{42}$ Apel, Hermeneutics, p. 476.

${ }^{43}$ Ibid., p. 478.
} 
sión, interpretación y aplicación), quedaria sin responder la pregunta por la validez de dicho proceso. En otras palabras, indicaría simplemente cómo es posible una comprensión, sea esta mala, regular o buena. Sin embargo, cuando, por ejemplo, nos abocamos a la interpretación de textos, no solo ejecutamos diversas lecturas, sino que reclamamos para ellas, al menos, cierto grado de plausibilidad. En la interpretación de Apel, los propios conceptos acuñados por Gadamer tienen no solo una pretensión descriptiva, esto es, "mostrar" cómo es posible la comprensión, sino que tienen también una pretensión normativa. En particular, Apel se refiere a la "anticipación de la perfección", el "círculo hermenéutico" y la "lógica de la pregunta y la respuesta", y aquellos conceptos que son rehabilitados contra la tradición cartesiana e ilustrada tales como sensus communis, prejuicios, autoridad y el concepto de lo clásico. La anticipación de la perfección, por ejemplo, "tiene que ser entendida como no ambigua e incuestionable en el sentido mínimo de una prioridad heurístico-metodológica del interpretandum ante los prejuicios posibles enfrentados (opposed) del intérprete" 44 . Se refiere, entonces, "al interés (concern) y la pretensión de verdad y también a su consistencia de sentido y coherencia"45.

En nuestro contexto, el concepto de mayor relevancia es el de la lógica de la pregunta y la respuesta. El principio fundamental reside aquí en que los enunciados o tesis filosóficas o de las ciencias del espíritu en general son respuestas a preguntas históricamente determinadas. Dicho de otra manera, los problemas de la filosofia no se encuentran en un "tercer mundo" (Popper) o son "eternos" e independientes, como pensaban los neokantianos. La pregunta es lógica y ontológicamente anterior a las respuestas, entendidas como tesis o proposiciones filosóficas. Sin embargo, nuevamente Apel insiste aquí en la instancia o problemática crítica. Es decir, si es cierto que los prejuicios, como la pre-comprensión del intérprete que abre una determinada pregunta, son una condición necesaria de posibilidad de la comprensión, Apel se pregunta si no es posible también sacar a la luz los prejuicios del texto, en el sentido de las preguntas e intereses que yacen detrás de él. Para Apel, es injustificada la prioridad que la hermenéutica filosófica otorga al interpretandum (el texto), dado que el proceso hermenéutico no es solo un acontecimiento temporal (transmisión de la tradición) sino que requiere de una instancia dialógica, una confrontación argumentativa.

\footnotetext{
${ }^{44}$ Apel, K-O., "The Hermeneutics of Being (Heidegger, Gadamer) Versus Transcendental Hermeneutics or Transcendental Pragmatics (Apel)", p. 749.

${ }^{45}$ Ibid., p. 479.
} 
Apel señala además una deficiencia o limitación de Gadamer respecto de la crítica al postulado del comprender mejor. En efecto, Gadamer distingue dos formas del "comprender mejor". La primera se encontraria en Kant y Fichte, y la segunda en Schleiermacher. Según Apel, es sobre todo contra esta última versión que Gadamer confronta, a saber: contra la idea de reproducción del proceso productivo de un autor (el genio). Ahora bien, en Kant y en Fichte el progreso es entendido como una idea regulativa referida al contenido semántico o la cosa de un texto, y no a la intención de un autor. Pero, "sorpresivamente", afirma Apel, "Gadamer condena ambas versiones"46. La rehabilitación del postulado del progreso de la comprensión de Apel, basado fundamentalmente en Kant, parte precisamente de la rehabilitación de la situación dialógica actual (en el presente de interpretación), que es donde podemos encontrar, por reflexión trascendental, el criterio para una hermenéutica-normativa. Gadamer alcanzó a rozar esta cuestión al colocar la "distancia en el tiempo" como la que permitiría separar los "verdaderos" prejuicios (por los cuales entendemos) de los "falsos" prejuicios (por los cuales malentendemos). Sin embargo, a pesar de realizar esta diferenciación normativa, no establece ningún criterio claro para efectuar tal distinción y, con ello, defender la posibilidad del progreso del comprender. Según Apel, esta deficiencia se debe a tres prejuicios de Gadamer, los cuales se encuentran interconectados:

1. El prejuicio, proveniente de Heidegger, de la temporalidad o historia del ser, según el cual una determinada apertura de sentido, lingüísticamente constituida, "determina en el modo de un destino los presupuestos de posibles juicios -verdaderos y falsos- desde el comienzo"47. Esta es la tesis que sostiene Lafont cuando dice que (en Heidegger) "el significado determina la referencia"48. Frente a ello, Apel defiende una posición, que según él tiene la forma del círculo hermenéutico, en la que se da una determinación recíproca (precisamente, "circular") entre las aperturas de sentido y procesos de aprendizaje y argumentos racionales.

2. El prejuicio, también proveniente de Heidegger, de la facticidad del ser en el mundo, en el sentido de "que todos los prejuicios que hacen posible la comprensión pertenecen ellos mismos a la tradición a ser posteriormente desarrollada... No hay prejuicios que, como un a priori

\footnotetext{
${ }^{46}$ Ibid., p. 751.

${ }^{47}$ Ibid., p. 754.

${ }^{48}$ Lafont, C., Lenguaje y apertura de mundo. El giro lingüistico de la hermenéutica de Heidegger, Madrid: Alianza Editorial, 1997.
} 
racional, hagan posible el diálogo hermenéutico con la tradición como un diálogo argumentativo. Esto es, no hay presupuestos racionales, independientes de la historia, de la hermenéutica" ${ }^{\text {"9. }}$

3. El prejuicio de la imposibilidad de una fundamentación de la dimensión normativa de la hermenéutica, a través de algo así como un principio trascendental del discurso hermenéutico.

En cuanto al primer punto, desde mi punto de vista, Apel no explica de un modo claro cómo sería esta relación circular entre "aperturas de sentido" y "procesos de aprendizaje". En todo caso, pareciera que es más bien cierta apertura de sentido lo que permite un proceso de aprendizaje empírico. Así, por ejemplo el concepto de "simultaneidad" en la física de Einstein (un ejemplo de avance de la ciencia que Apel cita a menudo) es más una forma de ampliación de sentido por vía de un análisis "semántico", mediante experimento mental. Los procesos empíricos de aprendizaje, como sostendría el propio Apel, presuponen el acuerdo (Verständigung), es decir presuponen la dimensión hermenéutica de la comunicación basada en un lenguaje intersubjetivamente válido que no es otro que el lenguaje fáctico e histórico determinado. Ahora bien, ¿cómo es posible entonces la "corrección" o transformación del sentido ya constituido? A mi juicio, la "experiencia" empuja los límites del lenguaje, por así decir, "desde adentro" pero, por experiencia no se puede entender meramente la experiencia "empírica". La experiencia estética, religiosa, intercultural (especialmente entre formas de vida muy diversas), y también la científica (cuando se trata de enfrentarse a "una materia extraña"), "provoca" al lenguaje. Sin embargo, se trata de una experiencia en y desde el lenguaje ${ }^{50}$.

En cuanto al segundo punto, la reflexión sobre la estructura dialógica conduce a una descripción de los presupuestos trascendentales de la argumentación que desarrolla la pragmática trascendental. Y respecto del tercero, para Apel la racionalidad ética se encuentra a la base de la racionalidad hermenéutica, la cual se encuentra a su vez a la base de todo conocimiento científico y pre-científico, y se expresa en la ética discursiva. Aquí se puede ver la articulación (arquitectónica) entre la hermenéutica trascendental (reflexión sobre la constitución o apertura de sentido), la pragmática trascendental (reflexión sobre la justificación de la validez) y la ética discursiva (en cuanto teoría moral).

\footnotetext{
49 Ibid.

50 De algún modo, lo que estoy tratando de decir es que no es necesario "refutar" el holismo del significado o apelar a cierto "naturalismo" para "garantizar" el progreso del conocimiento científico.
} 
Finalmente, para concluir este apartado, es importante destacar algunos comentarios que Apel realiza en este texto respecto de las consecuencias relativistas de la hermenéutica de Gadamer. Esto puede ser sintetizado en la defensa de Apel de una hermenéutica post-dogmática y una correlativa ética postconvencional $^{51}$. El rechazo de Gadamer de la filosofia reflexiva, o de la reflexión trascendental, conduciría a la hermenéutica filosófica a no poder distinguir la base fáctica de consenso del consenso como idea regulativa en una comunidad ideal de comunicación y, consecuentemente, a reducir la ética a una aplicación situacional (prudencial) dentro de un determinado ethos o tradición que, como tal, tiene una normatividad a priori sobre toda discusión de normas. La ética post-convencional, tal como es defendida por la ética discursiva, parte del reconocimiento de normas universalmente válidas por vía reflexiva sobre los presupuestos normativos del discurso argumentativo. Tales presupuestos, éticamente relevantes, no se encuentran sometidos a la temporalidad e historicidad (aunque por supuesto su "descubrimiento", su génesis histórica, es reconstruible históricamente) y es precisamente desde tales presupuestos normativos que puede juzgarse el contenido material de las diferentes normas tradicionales o convencionales. En el caso de conflictos normativos interculturales, como es cada vez más común en un mundo globalizado, la hermenéutica no podría responder más que apelando a las instancias valorativas propias de cada mundo. Desde la perspectiva de Apel, podría decirse entonces que la respuesta gadameriana a la posibilidad de una ética filosófica como mero ejercicio de la phrónesis no es coherente con el carácter cuasi-trascendental y normativo que el propio Gadamer reclama para la estructura dialógica de la hermenéutica.

\section{A modo de conclusión}

En este trabajo he intentado situar tanto textual como problemáticamente las principales críticas de Apel a la hermenéutica filosófica. No he pretendido realizar un desarrollo sistemático de los posicionamientos de la ética discursiva, sino más bien proponer una sintesis de la lectura apeliana de Gadamer. Esta tarea es necesaria dado que, como dije al comienzo, Gadamer aparece como un interlocutor casi permanente en la filosofia de Apel y no existen hasta el momento estudios que se ocupen exhaustivamente de esta relación. Por otra parte, las referencias que se pueden encontrar en la literatura especializada

\footnotetext{
${ }^{51}$ Apel, K-O., Diskurs und Verantwortung. Das Problem des Übergangs Poskonventionellen Moral, Fráncfort d.M.: Suhrkamp, 1988.
} 
se ocupan casi exclusivamente de la discusión en torno a la hermenéutica y la crítica de las ideologias; discusión que, como he intentado mostrar, en cierto modo queda a un costado en los textos desde la década de 1980 en adelante. Para mostrar esto, entonces, propuse analizar tres trabajos de Apel sobre la hermenéutica y en especial sobre Gadamer. Los tres textos permitieron mostrar diferentes aristas de esta relación con la hermenéutica. En primer lugar, la propuesta apeliana de un concepto de comprensión como idea regulativa; en segundo lugar, la idea de progreso que tal idea regulativa entraña; y, en tercer lugar, la defensa de la filosofia basada en el entendimiento dialógico, en lugar de (o en contra de) una filosofia basada en el acontecimiento y las consecuencias que tal principio supone para la filosofia práctica. Estos tres elementos pueden insertar a la concepción apeliana de la hermenéutica dentro de una tradición hermenéutica ilustrada. Como ha señalado Jean Greisch, "el hecho de que podamos elegir dos entre dos posibles lecturas de la historia de la hermenéutica incide sobre la idea que uno se hace de sus tareas futuras"52. Esas dos lecturas remiten a dos trayectorias: una, la más conocida, remite a Schleiermacher y Dilthey, su "expresión fenomenológica" en la hermenéutica de la facticidad de Heidegger hasta "florecer" con Gadamer; otra, en cambio, mucho menos conocida, remite al siglo XVIII, nada menos que al Siglo de las Luces y cuenta a Dannhauer como uno de sus mayores exponentes. Es en esta segunda trayectoria donde podemos encontrar el antecedente y el impulso para un retorno de la ontología a la epistemología, de la verdad como acontecimiento a la verdad como validez y de la comprensión infinita a la idea regulativa del comprender mejor. Como se ha visto a lo largo de este trabajo este postulado del "comprender mejor" de algún modo sintetiza la crítica y la propuesta de Karl-Otto Apel a la hermenéutica filosófica de Gadamer, y es, desde un punto de vista histórico, una concepción entendida en términos de Ilustración. Lejos del romanticismo, el postulado del comprender mejor de Apel bien podría ser formulado como ya lo hizo Wolff: "Leemos libros para aprender a conocer lo que se encuentra escrito en ellos. Pero esto requiere 1) que comprendamos correctamente al autor, 2) que captemos bien las cosas expuestas" 53 .

Recibido: $17 / 06 / 2016$

Aprobado: 16/02/2017

\footnotetext{
${ }^{52}$ Greisch, J., El cogito herido. La hermenéutica filosófica y la herencia certesiana, Buenos Aires: UNSAM, 2001, p. 86.

53 Citado en Greisch, J. o.c., p. 104.
} 


\section{Bibliografia}

Apel, K.-O., "Das Verstehen (eine Problemgeschichte als Begriffsgeschichte)", en: Archiv für Begriffsgeschichte, v. I, 1955, pp. 142-199.

Apel, K.-O. Die Idee der Sprache in der Tradition des Humanismus von Dante bis Vico, Bonn: Bouvier, 1975 [1963].

Apel, K.-O., Die Erklären:Verstehen-Kontroverse in transzendentalpragmatischer Sicht, Fráncfort d.M.: Suhrkamp, 1979.

Apel, K.-O., Understanding and Explanation. A Transcendental-Pragmatic Perspective, MIT Press, 1984.

Apel, K.-O., La transformación de la filosofía, Madrid: Taurus, 1985.

Apel, K.-O., Diskurs und Verantwortung. Das Problem des Übergangs Poskonventionellen Moral, Fráncfort d.M.: Suhrkamp, 1988.

Apel, K.-O., Auseinandersetzungen in Erprobung des transzendentalpragmatischen Ansatzes, Fráncfort d.M: Suhrkamp, 1998a.

Apel, K.-O., From a Transcendental-semiotic Point of View, Manchester: Manchester University Press, 1998b.

Apel, K.-O., "El desafío de una crítica total de la razón y el programa de una teoría de los tipos de racionalidad", en: Anales de la Cátedra Fracisco Suárez, n. 29, 1989, pp. 63-95.

Apel, K.-O., Semiótica transcendental y filosofía primera, Madrid: Síntesis, 2002.

Apel, K.-O., "The Hermeneutics of Being (Heidegger, Gadamer) Versus Transcendental Hermeneutics or Transcendental Pragmatics (Apel)", en: The Routledge Companion to Twenty Century Philosophy, Londres/Nueva York, Routledge, 2008, pp. 736-783.

Apel, K.-O., Semiótica filosófica, Buenos Aires: Prometeo Libros, 2009.

Apel, K.-O., Paradigmas de filosofía primera, Buenos Aires: Prometeo, 2013.

Apel, K-O. y Dussel, E., Ética del discurso y ética de la liberación, Madrid: Trotta, 2005.

Blanco Fernández, D., Pérez Tapias, J. y L. Sáez Rueda (eds.), Discurso y realidad. En debate con K.-O. Apel, Madrid: Trotta, 1994.

Böhler, D., "Philosophische Hermeneutik und hermeneutische Methode", en Fuhrmann, M. y otros (eds.), Text und Applikation, Múnich: Wilhelm Fink Verlag, 1981, pp. 483-511.

Böhler, D., "Transzendentalpragmatik und kritische Moral. Über die Möglichkeit und die moralische Bedeutung einer Selbstaufklärung der Vernunft", en: Kuhlmann, W. y D. Böhler (eds.), Kommunikation und Reflexion. Zur Diskussion der Transzendentalpragmatik Antworten auf Karl-Otto Apel, Fráncfort d.M.: Suhrkamp, 1982.

Borrelli, M., "De la hermenéutica del ser de Martin Heidegger, a la hermenéutica trascendenttal de Karl-Otto Apel”, en: Fornari, A., Pérez Zavala, C. y J. Wester (comps.), La razón en tiempos difíciles. Homenaje a Dorando Michelini, Río Cuarto: Ediciones del ICALA, 2010, pp. 63-73.

Crelier, A., De los argumentos trascendentales a la hermenéutica trascendental, La Plata: Edulp, 2010. 
Damiani, A., "Humanismo civil y hermenéutica filosófica. Gadamer lector de Vico", en: Cuadernos sobre Vico 15-16, 2010, pp. 31-47.

Ferraris, M., Historia de la hermenéutica, México: Siglo XXI, 2002.

Gadamer, H.-G., Verdad y método II, Salamanca: Sígueme, 1998.

Gadamer, H.-G., Verdad y método, Salamanca: Sígueme, 1999.

Greisch, J., El cogito herido. La hermenéutica filosófica y la herencia cartesiana, Buenos Aires: UNSAM, 2001.

Grondin, J., Introducción a la filosofia hermenéutica, Barcelona: Herder, 1999.

Grondin, J., Introducción a Gadamer, Barcelona: Herder, 2003.

Kuhlmann, W., "Los argumentos reflexivos, ¿son solo triquinuelas?", en: Fornari, A., Pérez Zavala, C. y J. Wester (comps.), La razón en tiempos dificiles. Homenaje a Dorando Michelini, Río Cuarto: Ediciones del ICALA, 2010, pp. 49-62.

Lafont, C., Lenguaje y apertura de mundo. El giro lingüístico de la hermenéutica de Heidegger, Madrid: Alianza Editorial, 1997.

Mendieta, E., The Adventures of Transcendental Philosophy: Karl-Otto Apel's Semiotics and Discourse Ethics, Rowman \& Littlefield, 2002.

Scivoletto, G., "El impacto de Heidegger y Gadamer en la hermenéutica trascendental de Apel", en: Contrastes. Revista Internacional de Filosofia, v. XX, n. 1, 2015, pp. 47-66. https://doi.org/10.24310/contrastescontrastes.v20i1.2296 